\title{
LISTE DES RÉDACTEURS
}

Astruc Didier, professeur à l'université Bordeaux 1, membre de I'Institut universitaire de France.

BARDEZ Élisabeth, professeur des universités, Conservatoire national des arts et métiers et ENS de Cachan, UMR CNRS 8531, IFR d'Alembert.

BERNIER Jean-Claude, professeur émérite de l'université Louis Pasteur, Strasbourg.

BLONDEL-MÉgrels Marika, historienne de la chimie, Institut d'histoire et de philosophie des sciences et des techniques, CNRS/Paris I.

BRIOT Eugénie, doctorante au Centre d'histoire des techniques et de l'environnement, CNAM, ATER à l'université de Marne-la-Vallée.

Champeau-Fonteneau Virginie, maître de conférences en histoire des sciences et des techniques, GHDSO-EST, université Paris-Sud XI, Orsay.

ChezEAu Jean-Michel, professeur émérite à l'université de Haute-Alsace, ancien directeur de I'École nationale supérieure de chimie de Mulhouse, chercheur associé au CRESAT.

Chezeau Nicole, docteur en histoire des sciences et des techniques, chercheur associée au CRESAT, université de Haute-Alsace, Mulhouse.

CHRISTOPHE Laurent, ingénieur technico-commercial.

CHRISTOPHE Roger, historien des métiers et industries de la chimie.

Claro-Gomes José, docteur en histoire des sciences, université Paris X.

Clavaguera Simon, ingénieur-chimiste, doctorant, CEA Le Ripault, ENSCM.

DelHAES Pierre, directeur de recherches CNRS émérite, au Centre de recherche Paul Pascal, Bordeaux.

DesmaRescaux Philippe, ancien directeur général de Rhône-Poulenc, président-fondateur du Forum mondial des sciences de la vie : BioVision.

Dimitrov Omourtague, ingénieur ENSCP, docteur ès sciences, ancien directeur de recherches au CNRS.

Dubar Jacques (décédé le 28 mars 2006), ancien élève de l'École polytechnique, docteur ès sciences.

EMPTOZ Gérard, docteur $3^{\mathrm{e}}$ cycle (chimie organique), docteur ès sciences physiques, professeur émérite de l'université de Nantes, historien de l'innovation technologique (chimie, combustibles, énergie, brevets d'invention).

Esbelin Christian, Development Scientist, Huntsman Polyurethanes, Everberg, Belgique. 
Faudue Danielle, docteur en histoire des sciences, chercheur associée au Groupe d'histoire et de diffusion des sciences d'Orsay (GHDSO), université Paris-Sud Xl, Orsay.

FILLEt Pierre, a été directeur de la recherche chimique de Rhône-Poulenc, délégué général du CADAS de l'Académie des sciences. Membre de l'Académie française des technologies.

FLAHAUT Jean, doyen honoraire de la faculté de pharmacie, président honoraire de l'Académie de pharmacie, président honoraire de la Société d'histoire de la pharmarcie.

FOURNIER Josette, professeur honoraire de l'université d'Angers en chimie organique, auteur d'une cinquantaine de publications en histoire des sciences.

GaY-Belulte Alain, président de l'Union nationale des Associations françaises d'ingénieurs chimistes. Ingénieur ENSCCF de la faculté des sciences de Lyon.

HURWIC Anna.

HuYNH Renaud, directeur, musée et archives de I'Institut du radium, UMS 6425 CNRS/Institut Curie, Paris. www.curie.fr/musee.

Jaussaud Philippe, université Lyon 1, EA 4148, LEPS, Villeurbanne.

KouneLIS Catherine, responsable de la bibliothèque et du centre de ressources historiques de I'ESPCl, historienne de la chimie.

LAFFITE Marc, docteur ès sciences, ancien président de la commission de Thermodynamique, ancien membre de la division de chimie physique, ancien membre de la commission Enseignement de I'IUPAC.

LAIRE Robert de.

LAMY Denis, ingénieur de recherche au CNRS, UMS 2700, Muséum national d'histoire naturelle, département Systémique et Évolution, Paris.

LANGLINAY Erik, professeur agrégé d'histoire, doctorant EHESS.

LATTES Armand, professeur émérite à l'université Paul Sabatier, membre de l'Académie nationale de pharmacie.

LeSTEL Laurence, chercheur au Centre d'histoire des techniques et de l'environnement, CDHTE-CNAM.

LETTÉ Michel, chercheur et enseignant en histoire des sciences et des techniques au Conservatoire national des arts et métiers (CDHTE-CNAM).

MAZIĖRES Marie-Rose, chercheur CNRS à I'Université Paul Sabatier, Toulouse, UMR 5068, Synthèse et physicochimie de molécules d'intérêt biologique.

MıLot Claude, professeur, UMR CNRS-UHP 7565, équipe de chimie et biochimie théoriques, université Henri Poincaré, Nancy.

Pacault Adolphe, Directeur honoraire du centre de recherche Paul Pascal, professeur émérite à l'université de Bordeaux 1, membre correspondant de l'Académie des sciences.

Patard Louis, docteur en chimie.

Pigeard-Micault Natalie, docteur en histoire des sciences, coordinatrice de Gallica pour les sciences et les techniques à la BnF.

Poisson Jacques, professeur honoraire à la faculté de pharmacie de Châtenay-Malabry, université Paris-Sud XI.

RignY Paul, physico-chimiste, ancien du CEA, du CNRS et de l'ANDRA. 
RIVAlL Jean-Louis, professeur émérite de chimie théorique à l'université Henri Poincaré, Nancy.

RuLLIĖRE Sonia.

SILLION Bernard, docteur, vice-président de la SFC.

TACHOIRE Henri, professeur émérite de l'université de Provence, secrétaire perpétuel de l'Académie des sciences, lettres et arts de Marseille.

TEISSIER Pierre, physico-chimiste de formation, il termine aujourd'hui une thèse sur l'histoire de la chimie du solide en France (1950-2000).

Tomic Sacha, agrégé de sciences-physiques, docteur en histoire des sciences, enseignant associé au Service d'histoire de l'éducation (INRP-ENS).

TRAVERS Christine, docteur en sciences pétrolières, professeur et directeur du centre Raffinage-Pétrochimie-Gaz à l'ENSPM.

Viel Claude, professeur honoraire à la Faculté de pharmacie de Tours, ancien président du Club d'histoire de la chimie de la SFC, vice-président de l'Académie de Touraine. 
This page intentionally left blank 